\title{
ON LOCAL AND UNIFORMLY LOCAL TOPOLOGICAL PROPERTIES ${ }^{1}$
}

\author{
JAMES DUGUNDJI AND ERNEST MICHAEL
}

1. The main purpose of this paper is to show that certain properties of a metrizable space, such as local connectedness or local contractibility or $\mathrm{LC}^{n}$ or local compactness, hold uniformly with a properly chosen metric. ${ }^{2}$ This result, while easy to prove, seems to be new, and often permits simplifications in proofs dealing with various kinds of local connectedness. In $\S 2$, we obtain an analogous (actually stronger) result dealing with coverings.

Definition 1. An ordering ${ }^{3} \alpha$ of the subsets of a space $X$ is proper provided:

(a) If $W \alpha V$, then $W \subset V$;

(b) If $W \subset V$, and $V \alpha R$, then $W \alpha R$;

(c) If $W \alpha V$, and $V \subset R$, then $W \alpha R$.

DEFINITION 2. Let $\alpha$ be a proper ordering of the subsets of a metric space $X$. Then

(a) $X$ is locally of type $\alpha$ if, whenever $x \in X$ and $V$ is a neighborhood of $x$, then there exists a neighborhood $W$ of $x$ such that $W \alpha V$.

(b) $X$ is uniformly locally of type $\alpha$ if to every $\epsilon>0$ there corresponds a $\delta>0$ such that $S_{\delta}(x) \alpha S_{\epsilon}(x)$ for every $x \in X{ }^{4}$

For example, if $W \alpha V$ means that every pair of points in $W$ is contained in a connected subset of $V$, then locally of type $\alpha$ means locally connected. If $W \alpha V$ means that $W$ is contractible in $V$, then locally of type $\alpha$ means locally contractible. Other topological properties which arise in this manner are $n$ - LC (where $W \alpha V$ means that every continuous mapping of the $n$-sphere into $W$ is homotopic to a constant mapping in $V$ ), $\mathrm{LC}^{n}$ (i.e., $k$-LC for all $k \leqq n$ ), and analogous kinds of homological connectedness.

Presented to the Society, April 30, 1955; received by the editors March 7, 1955 and, in revised form, May 10, 1955.

1 This paper was worked out while the second author was an A.E.C. Fellow.

2 For local connectedness, this was proved by a simple direct method in [5]. For local compactness, this is implicit in [4], and also follows directly from [2, Corollary 2.3].

${ }^{3}$ By (a) and (b), this "ordering" is transitive, but it need not satisfy any of the other order-axioms.

'If $X$ is a metric space with metric $\rho$, then $S_{f}(x)_{0}^{\circ}\left(=S_{e}^{\rho}(x)\right)$ denotes $\left\{\left.x^{\prime} \in X\right|_{\rho}\left(x, x^{\prime}\right)\right.$ $<\}$. 
THEOREM 1. If $\alpha$ is a proper ordering on the subsets of a metrizable space $X$, and if $X$ is locally of type $\alpha$, then there exists a metric $\rho$ on $X$, agreeing with the topology, such that $(X, \rho)$ is uniformly locally of type $\alpha$. Moreover, if $\sigma$ is any metric on $X$ agreeing with the topology, then we can choose $\rho$ to generate a finer uniform structure than $\sigma .^{5}$

Proof. Let $\sigma$ be any metric on $X$ which agrees with the topology. Since every metric space $X$ is fully normal $[3$, p. 52, Theorem 8.12] (i.e., every open covering of $X$ has an open $\Delta$-refinement $t^{6}$ ), we can (by Definition 1) inductively construct a sequence $\left\{V_{n}\right\}_{n=1}^{\infty}$ of open coverings of $X$ (starting with an arbitrary $U_{1}$ ) such that, for every $n \geqq 1$,

(a) $V_{n+1}$ is a $\Delta$-refinement of $V_{n}$,

(b) If $V \in V_{n+1}$, then $V \alpha W$ for some $W \in V_{n}$,

(c) If $V \in V_{n}$, then $\sigma$-diam $(V)<2^{-n}$.

By (a) and a theorem of Tukey [3, p. 51, Theorem 7.4], we can now find a continuous pseudometric $\rho$ on $X$ which is equivalent to $\left\{V_{n}\right\}_{n=1}^{\infty}$; i.e., for every $\epsilon>0$ there exists an $n$ such that $V_{n}$ is a refine-

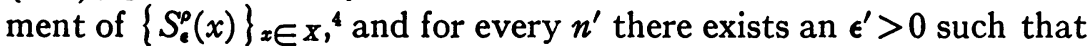
$\left\{S_{e^{\prime}}^{\prime}(x)\right\}_{x \in X}$ is a refinement of $V_{n^{\prime}}$. Hence, by (c), $\rho$ generates a finer uniform structure than $\sigma$, and hence $\rho$ is actually a metric. Finally, (b) implies that $X$ is uniformly of type $\alpha$ with respect to $\rho$. This completes the proof.

CoROLLARY 1. If $\alpha$ is a proper ordering of the subsets of a compact metric space, and if $X$ is locally of type $\alpha$, then $X$ is uniformly locally of type $\alpha$.

Proof. This follows immediately from Theorem 1, and the fact that a compact space admits only one uniform structure.

We conclude this section by remarking that, as the proof shows, Theorem 1 remains true for all fully normal spaces $X$, provided "metric agreeing with the topology" is replaced by "continuous pseudometric" or by "uniform structure agreeing with the topology."

2. In this section, we shall indicate how the definitions and results of the first section can be generalized.

Definition $1^{\prime}$. An ordering ${ }^{3} \xi$ of coverings of a set $X$ is proper provided:

(a) If $\mathscr{W} \mho$, then $\mathscr{W}$ is a refinement of $\mho$;

'If one takes $\tau=\rho+\sigma$, then $\tau$ has all the properties of $\rho$ in Theorem 1, and moreover $r \geqq \sigma$.

- $W$ is a $\Delta$-refinement of $V$ if, for every $x \in X$, there exists a $V \in V$ such that $(U\{W \in W \mid x \in W\}) \subset$. 
(b) If $W$ is a refinement of $V$, and $V \xi R$, then $\mathscr{W}$;

(c) If $\mathscr{\xi} \mathcal{V}$, and $U$ is a refinement of $R$, then $W \xi R$.

REMARK 1 . If $\alpha$ is a proper ordering of the subsets of $X$, then $\alpha$ generates a proper ordering $\bar{\alpha}$ of the coverings of $X$ as follows: $W_{\bar{\alpha}} \mathcal{U}$ means that for every $W \in \mathscr{W}$ there exists a $V \in \mathcal{U}$ such that $W \alpha V$.

Definition $2^{\prime}$. Let $\xi$ be a proper ordering of the coverings of a metric space $X$. Then

(a) $X$ is of type $\xi$ if, for every open covering $U$ of $X$, there exists an open covering $\mathscr{W}$ of $X$ such that $\mathscr{W} \boldsymbol{U}$.

(b) $X$ is uniformly of type $\xi$ if, for every $\epsilon>0$ there exists a $\delta>0$ such that $\left[\left\{S_{\delta}(x)\right\}_{x \in X}\right] \xi\left[\left\{S_{\epsilon}(x)\right\}_{x \in X}\right]$.

REMARK 2. If $\alpha$ is a proper ordering of the subsets of a metric space $X$, and if $\bar{\alpha}$ is as in Remark 1 , then $X$ is (uniformly) locally of type $\alpha$ if and only if $X$ is (uniformly) of type $\bar{\alpha}$.

There are proper orderings of the coverings of a metric space $X$ which cannot be generated, in any obvious manner, by a proper ordering on the subsets of $X$. For instance, we can define $\mathscr{U} \zeta$ by "every partial realization of a simplicial CW-complex into $X$ relative to $\mathcal{W}$ can be extended to a full realization relative to $U$." (As observed in $[1$, p. 358], a metric space is of this type $\zeta$ if and only if it is an ANR (metric)). An example, kindly suggested by the referee, of a proper ordering of the coverings of a metric space $X$ which cannot in any manner be generated by a proper ordering of the subsets of $X$, is $\Delta$-refinement. For suppose this is of the form $\vec{\alpha}$. If now $U$ and $V$ are open subsets of $X$ with $\bar{V} \subset U$, then one easily constructs an open $\Delta$-refinement $U$ of the covering $\{U, X-\bar{V}\}$ such that $V \in V$; hence $V \alpha U$ or $V \alpha(X-\bar{V})$, and therefore $V \alpha U$. This implies that, if $\left\{V_{i}\right\}$ and $\left\{U_{i}\right\}$ are open coverings of a metric space, and if $\bar{V}_{i} \subset U_{i}$ for all $i$, then $\left\{V_{i}\right\}$ is a $\Delta$-refinement of $\left\{U_{i}\right\}$. But (unless $X$ is trivial) this is patently false, and we have a contradiction.

Our analogue of Theorem 1 for coverings is

TheOREM 1'. The same as Theorem 1, but stated for coverings instead of subsets.

Proof. The same as for Theorem 1.

\section{REFERENCES}

1. J. Dugundji, An extension of Tietze's theorem, Pacific Journal of Mathematics vol. 1 (1951) pp. 175-186.

2. E. Michael, Local properties of topological spaces, Duke Math. J. vol. 21 (1954) pp. $163-171$.

3. J. W. Tukey, Convergence and uniformity in topology, Annals of Mathematics Studies, no. 2, Princeton, 1940. 
4. H. E. Vaughan, On locally compact, metrisable spaces, Bull. Amer. Math. Soc. vol. 43 (1937) pp. 532-535.

5. G. T. Whyburn, A certain transformation on metric spaces, Amer. J. Math. vol. 54 (1932) pp. 367-376.

University of Southern California ANd UNIVERSITY OF WASHINGTON

\section{A THEOREM OF ELIE CARTAN}

G. A. HUNT

André Weil [1] and Hopf and Samelson [2] have given a topological proof of the following theorem of Elie Cartan.

Two maximal Abelian subgroups of a compact connected Lie group $G$ are conjugate within $G$.

I present a simple metric proof.

Lemma. If $x$ and $y$ are elements of the Lie algebra $g$ of $G$ then $\left[x, A_{\sigma} y\right]$ vanishes for some inner automorphism $A_{\sigma}$ of $G$.

Proof. Because $G$ is compact one can define on $g$ a nonsingular bilinear form $(u, v)$ which is invariant: $([u, v], w)+(v,[u, w]) \equiv 0$. We choose $\epsilon$ in $G$ so that $\left(x, A_{\sigma} y\right)$ attains its minimum for $\sigma=\epsilon$; without loss of generality we may assume $\epsilon$ to be the neutral element of $G$, and then $A_{\mathfrak{c}} y=y$. If now $z$ is any element of $g$ the function $\left(x, A_{\exp (t z)} y\right)$ has a minimum for $t=0$, so that its derivative vanishes there. Thus, keeping in mind that

$$
\left.\frac{d}{d t} A_{\exp (t z)} y\right|_{t=0}=[z, y],
$$

we have $(x,[z, y])=0$. From this equation and from the invariance of the bilinear form it follows that $([x, y], z)=0$ for all $z$; this can happen only if $[x, y]$ vanishes, for the bilinear form is nondegenerate.

Before proving Cartan's theorem I recall some well-known facts: A maximal Abelian subgroup $\mathfrak{H C}$ of $G$ is a torus group; there is an element $x$ in the Lie algebra $\mathfrak{h}$ of $\mathcal{H C}$ such that the one parameter group $\exp t x$ is dense in $\mathfrak{H C}$; if $y$ belongs to $g$ and $[x, y]=0$, then $y$ must lie in $\mathfrak{h}$.

Matters being so, let $\mathcal{J C}^{\prime}$ be a second maximal Abelian subgroup of $G$ and $x^{\prime}$ an element of its Lie algebra bearing the same relation

Received by the editors May 20, 1955. 